\title{
Qualitative changes in the proteome of extracellular vesicles accompanying cancer cell
}

\section{transition to mesenchymal state}

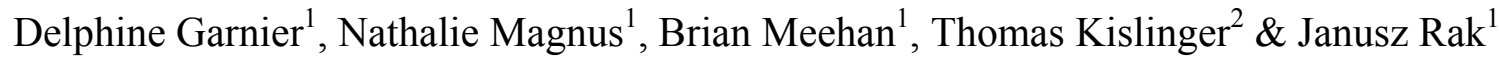

${ }^{1}$ Montreal Children's Hospital, RI MUHC, McGill University, Montreal, Quebec, Canada

${ }^{2}$ Ontario Cancer Institute, Toronto, Ontario, Canada

Corresponding author:

Janusz Rak

Montreal Children's Hospital Research Institute, McGill University,

Place Toulon, 4060 Ste Catherine West, PT-232

Montreal, QC, H3Z 2Z3, Canada

Tel: (514) 412-4400 ext 22342

Fax: (514) 412-4331

e-mail: janusz.rak@mcgill.ca

Short title: EMT, proteomic, extracellular vesicles

Total Word Count: 4622

Abstract Word Count: 196

Figure count: 5

Reference count: 65

Abbreviations: EGFR, Epidermal Growth Factor Receptor; EMT, Epithelial-to-Mesenchymal Transition; EVs, Extracellular Vesicles; TGF $\alpha$ - Transforming Growth Factor alpha. 


\begin{abstract}
Transitions of the cancer cell phenotype between epithelial and mesenchymal states is likely to alter the patterns of intercellular communication. In this regard we have previously documented that EMT-like changes trigger quantitative changes in exosomal vesicle emission in A431 cancer cells driven by oncogenic epidermal growth factor receptor (EGFR). Here we report that extracellular vesicles (EVs) produced by these cancer cells in their epithelial and mesenchymal states exhibit profound qualitative differences in their proteome. Thus, induction of the EMT-like state through blockade of E-cadherin and EGFR stimulation resulted in mesenchymal shift in cellular morphology and enrichment in the CD44-high/CD24-low immunophenotype, often linked to cellular stemness. This change also resulted in reprogramming of the EV-related proteome (distinct from that of corresponding cells), which contained 30 unique protein signals, and revealed enrichment in pathways related to cellular growth, cell-to-cell signalling, and cell movement. Some of the most prominent EV-related proteins were validated, including integrin $\alpha 2$ and tetraspanin CD9. We propose that changes in cellular differentiation status translate into unique qualitative rearrangements in the cargo of EVs, a process that may have implications for intercellular communication and could serve as source of new biomarkers of EMT-like changes in cancer.
\end{abstract}




\section{Introduction}

Epithelial-to-Mesenchymal Transition (EMT) is a cellular transdifferentiation program, whereby cells lose their epithelial attributes and assume features of the mesenchymal lineage, along with elongated morphology, increased motility, reduced proliferation rate and expression of marker proteins, such as vimentin. This process occurs normally at certain stages of embryonal development, but is also reinstated during cancer progression, where it signifies acquisition of increased invasiveness and tumor cell stemness [1-3].

Mesenchymal phenotype can be induced in epithelial cancer cells by several stimuli, including activation of certain proto-oncogenes (Ras) and growth factor receptor pathways, such as those driven by transforming growth factor beta (TGF $\alpha$ ), hepatocyte growth factor (HGF) receptor (MET), or epidermal growth factor receptor (EGFR). This change may also result from environmental cues, including hypoxia, or occur as a result of other influences that can change the activity of EMT-regulating transcription factors, such as Twist, YB-1, Snail, Brachyury and several others $[1,4,5]$.

One of the consistent hallmarks of EMT is the disruption of adherens intercellular junctions, which liberates the cells from spatial and signalling constraints, and may enable their greater motility. This process is often linked with downregulation of E-cadherin [1], which is a part of multimolecular hubs that modulate the activity of growth factor pathways, including EGFR [6]. 
Notably, disruption of E-cadherin-mediated adhesion by RNA interference [7], or usage of specific neutralising antibodies [8] has been reported to induce EMT-like features in epithelial cancer cells.

We have previously described an EMT-like phenotype associated with human squamous cell carcinoma cells (SCC) in vivo, and in xenografts of the well characterized SCC-derived cell line, A431, which is driven by the amplification of the epidermal growth factor receptor (EGFR) [9]. Interestingly, these cells exhibit epithelial morphology in culture, but can be induced to undergo EMT-like changes in the presence of the E-cadherin neutralizing antibody (SHE78-7) and EGFR activating ligands, such as transforming growth factor alpha $(\mathrm{TGF} \alpha)[8,9]$. Under these conditions intercellular junctions become dissociated, and the cells assume elongated morphology, express vimentin and change their intracellular distribution of several proteins, including $\beta$-catenin [9]. This change is also associated with an increased proficiency at forming metastatic nodules in lungs, and an exaggerated proangiogenic and procoagulant phenotype, at least in part due to upregulation of tissue factor (TF) on the tumor cell surface $[8,9]$. Thus, EMT appears to alter not only intrinsic cellular properties of cancer cells, but also their ability to interact with the vascular system and their tissue surroundings in ways that are not fully understood.

Indeed, one of the most intriguing features associated with EMT, including in the case of A431 cells, is the change in the cellular secretome, especially as it relates to the emission profile of extracellular vesicles (EVs) [8-11]. EVs are membrane structures that various cells shed into 
their microenvironment, either constitutively, or as a result of stress, activation or oncogenic transformation [12-14]. There is a growing body of evidence to suggest that the uptake of cancerrelated EVs by various types of recipient cell impacts their properties and behaviour, and these changes have been implicated in such processes as angiogenesis, metastasis, immunoregulation, activation of the coagulation system, intercellular transfer of oncogenes and several other events [14-22].

The various biological activities and molecular composition of EVs depend on mechanisms of their biogenesis (vesiculation), of which there are at least two main pathways. Thus, EVs may be formed due to plasma membrane blebbing, which results in formation of larger vesicles known as ectosomes or microvesicles $(100-1000 \mathrm{~nm}$ in diameter). Smaller EVs (30-100nm), known as exosomes, can be generated by the endosomal pathway, and they exhibit distinct molecular and physical properties as well as cellular functions [23]. For example, exosomes have been implicated in the regulation of cell adhesion [24], pericellular proteolysis [25], removal of superfluous molecules from differentiating cells [26], attenuation and modulation of intracellular signalling cascades [9,27-29] and formation of regulatory gradients of morphogens, such as Wnt [30].

Interestingly, vesiculation is known to lead to selective enrichment of some bioactive molecules but not others in the EV cargo, and the nature, mechanisms and consequences of these combinatorial processes encompassing large numbers of molecular species involved are still poorly understood $[21,31,32]$. 
In this regard the nexus between EMT and the resulting changes in cellular vesiculation profiles has been explored by only a handful of recent studies [9-11,11]. For example, we have recently reported quantitative changes in the vesiculation pattern of mesenchymal A431 cells, with the prominent emission of procoagulant exosomes [9]. In the present study we asked whether these changes affect the qualitative and quantitative properties of tumor-related EVs, in particular, whether they alter the composition of the vesicular proteome. Indeed, we found that, as A431 cells transit between epithelial and mesenchymal states, the protein cargo of their derived EV undergoes a profound rearrangement. This shift was neither an exact replica of underlying changes within the proteome of EV-producing cells, nor a simple summation of the individual effects of EMT-inducers, such as the E-cadherin blocking antibody and EGFR agonists. Rather, at least $30 \mathrm{EV}$-associated protein changes were unique to the mesenchymal phenotype of their producing cells. In addition, the pathway analysis of the EV-related proteome revealed that mesenchymal transition predominantly affected proteins involved in proliferation, communication, junction formation, ECM adhesion and vesiculation itself, which is consistent with the nature of the underlying biological processes. We postulate that the composition of EVs may, in principle, serve as a biomarker of processes involved in cancer cell transdifferentiation, and may also define the biological role and activity of EVs in these settings.

\section{Material and Methods}

Cell culture and treatments 
The human squamous cell carcinoma (SCC) A431 cell line was obtained from American Type Culture Collection (ATCC, Manassas, VA, USA) and cultured in DMEM (Life Technologies, Grand Island, NY, USA) supplemented with 10\% fetal bovine serum (FBS)(Multicell Wisent Inc., St-Bruno, QC, Canada) depleted of EVs. Cells were serum-starved overnight, prior to treatments with $50 \mathrm{ng} / \mathrm{mL}$ of TGF $\alpha$ (Life Technologies), $2 \mu \mathrm{g} / \mathrm{mL}$ SHE78-7 anti-E-cadherin neutralizing antibody (Life Technologies) for $48 \mathrm{~h}$.

\section{Preparation of extracellular vesicles}

EVs were obtained by ultracentrifugation as described earlier [9,14,33,34]. Briefly, culture supernatants were centrifuged for $10 \mathrm{~min}$ at $400 \mathrm{~g}$, followed by $2 \mathrm{~h}$ at $100,000 \mathrm{~g}$ to pellet EVs, which were washed extensively in phosphate buffered saline (PBS).

\section{Western blotting}

As described earlier [35], membranes were prepared and probed with the following primary antibodies: rabbit anti-integrin $\alpha 2$ (Abcam, Toronto, ON, Canada), rabbit anti-human $\gamma$-catenin (Abcam), mouse anti-CD9 (Santa Cruz Biotechnology, Dallas, TX, USA), mouse anti-Flotillin-1

(BD Biosciences, Mississauga, ON, Canada), rabbit anti-GAPDH (Abcam), mouse anti- $\beta$-actin (Sigma Chemical Co., St Louis, MO, USA). Horseradish peroxidase (HRP)-conjugated secondary antibodies (Dako, Mississauga, ON, Canada) and chemiluminescence (GE Healthcare, Piscataway, NJ, USA) were used for detection. 


\section{Immunofluorescence}

Cultured cells were fixed for $10 \mathrm{~min}$ in $4 \%$ paraformaldehyde, permeabilized with $0.1 \%$ Triton $\mathrm{X}-100$, and stained overnight at $4{ }^{\circ} \mathrm{C}$ with rabbit anti-human Integrin- $\alpha 2$ (Abcam) rabbit antihuman $\gamma$-catenin (Abcam), or FITC-phalloidin (Sigma) followed by their respective secondary Alexa Fluor antibodies (ㅍvitrogen).

Fluorescence-activated cell sorting

Single-cell suspensions were washed with phosphate-buffered saline containing $1 \%$ bovine serum albumin and $0.1 \%$ sodium azide before staining with FITC conjugated mouse anti-human CD44 antibody (BD Biosciences).

\section{Cell and EV lysis and protein extraction}

The cells were incubated in hypotonic lysis buffer containing 10mM HEPES, pH 7.4 for 30 minutes on ice. The suspension was briefly sonicated and Triton-X-100 was added to a final concentration of $1.5 \%$. The suspension was incubated at $4^{\circ} \mathrm{C}$ for 30 minutes, followed by centrifugation at $14,000 \mathrm{rpm}$ for 30 minutes at $4^{\circ} \mathrm{C}$. The supernatant was removed and used for proteomic analysis as previously described [36]. EVs were solubilised by incubation in $50 \mathrm{mM}$ ammonium bicarbonate $0.2 \%$ PPS detergent for 60 minutes at $37^{\circ} \mathrm{C}$, followed by digestion with trypsin as described below. 


\section{In-Solution Protein Digestion}

An aliquot of $150 \mu \mathrm{g}$ of protein from each cell lysate sample was precipitated overnight at $-20^{\circ} \mathrm{C}$ with 5 -volumes of ice-cold acetone, followed by centrifugation at $21,000 \mathrm{~g}$ for $15 \mathrm{~min}$. The protein pellet was solubilized in $8 \mathrm{M}$ urea, $2 \mathrm{mM}$ DTT, $50 \mathrm{mM}$ Tris- $\mathrm{HCl}, \mathrm{pH} 8.5$ at $37^{\circ} \mathrm{C}$ for 1 hour, followed by carboxyamidomethylation with $10 \mathrm{mM}$ iodoacetamide for 1 hour at $37^{\circ} \mathrm{C}$ in the dark. The samples were then diluted with $50 \mathrm{mM}$ ammonium bicarbonate, $\mathrm{pH} 8.5$ to $\sim 1.5 \mathrm{M}$ urea. Calcium chloride was added to a final concentration of $1 \mathrm{mM}$ and digested with a 1:25 molar ratio of recombinant, proteomics grade trypsin (Roche Diagnostics, Laval, QC) at $37^{\circ} \mathrm{C}$ overnight. The resulting peptide mixtures were solid phase-extracted with Varian OMIX cartridges (Mississauga, ON, Canada) according to the manufacturer's instructions and stored at $-80^{\circ} \mathrm{C}$ until further use.

Mass spectrometry (MS)

The samples were lysed and peptide mixtures were solid phase-extracted with Varian OMIX cartridges (Mississauga, ON, Canada). For analysis and protein identification, a fully automated 4-cycle, 8-h MudPIT procedure was set up similar as previously described (Taylor et al., 2009a) using a LTQ linear ion-trap (Fisher Scientific, Toronto, ON, Canada). Each sample was analyzed in duplicates. Raw files were converted to m/zXML using ReAdW and searched by a locally installed version of X!Tandem against a human UniProt protein sequence database as recently described [37]. To estimate and minimize our false positive rate the protein sequence database also contained protein sequence in its reversed amino acid orientation (target-decoy strategy). Search parameters were: Parent ion $\Delta$-mass of $4 \mathrm{Da}$, fragment mass error of $0.4 \mathrm{Da}$, and 
oxidation of methionine as a variable modification and IAA modification of cystein as a static modification (only for lysate fraction). A rigorous peptide quality control strategy was applied to effectively minimize false positive identifications, as previously described (Taylor et al., 2009b) $[$ FDR $<1 \%]$. To minimize protein inference we developed a database grouping scheme, and only report proteins with substantial peptide information, as recently reported (Sodek et al., 2008). Classification of proteins according to cellular pathways was performed with Ingenuity pathway analysis (IPA) software (Ingenuity Systems Inc., Redwood City, CA, USA). The experiments consisted of three runs, one pilot set and two full size sample sets the latter comprising all experimental conditions, in which untreated cells and cells treated with single agent served as reference, and these runs were manually spot checked for consistency with the pilot. To develop the protein profile associated with these treatments a cumulative approach was undertaken to overcome the impact of potential random sample-to-sample variability, which may result in missed signal for individual proteins. To accomplish this, both repeat samples from two independent experiments were combined and analysed, as per shotgun approaches described earlier by us and others $[38,39]$. 


\section{Results}

EVs secreted by A431 cells contain proteins of different origins and different functions. We have previously documented the ability of EGFR-driven human squamous cell carcinoma cells, A431, to emit extracellular vesicles of wide ranging sizes that can be visualized by electron microscopy. We have also shown that A431 cells undergo a mesenchymal change in phenotype, when exposed to the E-cadherin neutralizing monoclonal antibody (SHE78-7) and TGF $\alpha$, a potent EGFR agonist, a process that alters their profile of vesiculation (as schematically shown in Fig. 1A)[9]. This treatment induced a spinde shaped change in cell morphology, corresponding rearrangement in actin cytoskeleton (Fig. 1B-C) and provoked enrichment in the CD44-high/CD24-low immunophenotype, which is attributed to the stem-like population of cancer cells often linked with EMT [2,40], including the A431 cell line [41,42].

To understand the molecular underpinnings of EMT-related changes in A431 cell-derived EVs, we profiled their protein content using mass spectrometry (MS). Predictably, a large proportion of A431-derived EVs contained proteins annotated as either of membrane or cytoplasmic origin, with a sizeable and somewhat surprising contribution of nuclear signatures (Fig. 2A). Interestingly, marked differences were noted in the lists of the top 25 most abundant proteins identified in A431 EVs relative to the corresponding cell lysates (Table 1), which is consistent with the notion of selective rather than random assembly of the EV cargo [21].

We also used Gene Ontology (GO) classification to assess the global features of the EV proteome, of which a significant proportion was assigned to the metabolic process category (Fig. 
2B), with other prominent groupings involving binding (32\%) or catalytic activity (30\%)(Fig. 2BC). Amongst these proteins, $15 \%$ reveals a link to nucleic acid binding (Fig. 2D). While these are not processes normally associated with the biology of EV, the GO databases are shaped by what is known in the literature about various cellular protein groupings, and this may not necessarily reflect the most germane functions some of these proteins may have in the context of EVs.

Manifestation of the mesenchymal phenotype in the EV proteome. We next monitored the transition of A431 cells toward the mesenchymal state upon incubation with the anti-E-cadherin antibody, SHE78-7, combined with stimulation of EGFR using TGF $\alpha$ [9]. While each of these treatments alone and their combination (SHE78-7/TGF $\alpha$ ) exert dramatic effects on cellular behaviour, the corresponding cellular proteomes retain a significant level of overlap (655 in total for cells)(Figure 3A). The overlap also exists between the respective EV fractions (366 proteins commonly detected for the 4 different conditions; Figure 3B).

However, subsets of proteins were also found to be relatively specific for either control EVs (83), or EVs produced by SHE78-7 treated cells (37), EVs emitted under TGF $\alpha$ otreatment alone (12), or upon induction of EMT-like transition with both of these agents added simultaneously (30), as depicted in Figure 3B. Moreover our analysis of pathways represented by these EVrelated protein sets (IPA) revealed a significant enrichment in proteins representative of cellular growth and proliferation, cell-to-cell signalling and interaction, cellular movement, and cell 
morphology pathways, especially upon transition to the mesenchymal state (SHE78-7/TGF $\alpha$ ) as might be expected (Fig. 3D).

It is of interest that the degree of change involving these protein clusters were far less pronounced in cells then in their derived EVs (Fig. 3C). This may suggest that these proteins could exert their IPA-assigned functions in some recipient cells, to which they could be transferred by EVs, as recently suggested by a number of reports $[9,10]$. Alternatively, vesiculation may represent a mechanism to attenuate these particular protein functions by removing the respective molecular effectors from cells $[9,27]$.

Changes in abundance and composition of proteins in cells and their EVs. We observed that EMT triggered changes in both relative abundance and qualitative composition of proteins detected by our MS screens. These changes differed between cells and their corresponding EVs. Indeed, some proteins were quantitatively depleted in the EV fraction (e.g. polypyrimidine tract binding protein 1), while others were enriched in EVs (e.g. myosin-1b), as a function of EMT induction. These patterns are captured in the heatmap (Fig. 4), which lists proteins, for which there was more than 4 fold relative increase or decrease in spectral counts over the untreated controls (EVs or cells, respectively).

While the effects associated with the mesenchymal phenotype were of special interest, intriguing quantitative changes were also found in the case of treatment with TGF $\alpha$ or SHE78-7 alone (Fig. 4). As mentioned earlier, relatively few proteins, for which a major change in levels was recorded, exhibited similar patterns of increase or decrease between A431 cells and their 
corresponding EVs, at least in selected settings. The examples of those include: SPTAN1 (spectrin alpha), ST13 (suppression of tumorigenicity 13/HSP70 interacting protein) or HSD17B10 (17- $\beta$-Hydroxysteroid dehydrogenase X).

\section{Mesenchymal phenotype is associated with enrichment of EV-related proteins in integrins}

and cell junction proteins. Out of 32 proteins upregulated in EVs following the SHE78-7/TGF $\alpha$ treatment, by at least 2 fold (Table 2), 7 proteins (22\%) were a part of the vesicle-mediated transport mechanism, according to PANTHER protein classification. Moreover, tetraspanin CD9 and Alix (PDCD6IP), which are exosomal markers [43], were also enriched in EVs obtained from cells cultured under EMT-inducing conditions, compared to control EVs (Table 2). This observation is congruent with the exosomal peak observed in our previous experiments using nanoparticle tracking analysis (NTA) to measure the emission of exosomes following EMTinducing treatments (Garnier et al., 2012g).

When classified according to their GO Biological process annotation, more than half of these proteins were assigned to Cellular Process (GO:0009987), including 15 proteins related to cell communication (47\%), 12 to cell adhesion (37\%) and 6 proteins involved in cell motion (19\%). When these data were filtered according to signalling pathways, the integrin signalling, clearly, appeared as a predominant feature, with $22 \%$ of the 32 upregulated proteins being included. This is consistent with changes underlying both EMT and vesiculation processes, both of which involve adhesive events and the presence of integrins [24]. 
It is noteworthy that we were able to confirm a relatively selective integrin $\alpha 2, \gamma$-catenin and tetraspanin CD9 incorporation into the cargo of EVs, especially upon induction of the EMT-like state (Fig. 5A). Whereas integrin $\alpha 2$ and $\gamma$-catenin are prominently expressed in cancer cells, we only detected a very low level of CD9 in cellular lysates. This was consistent with the proteomic analysis, for which no CD9 signal was detected in the cellular fractions, and the same was true for immunostaining experiments with anti-CD9 antibody (data not shown). Naturally, this does not reflect the absence of CD9 in cells, a source of CD9 in EVs, but rather the propensity of this particular tetraspanin to exist parental cells via the vesiculation pathway [27]. On the other hand, immunofluorescent stainings of integrin $\alpha 2$, and $\gamma$-catenin documented a change in localization of these two proteins following mesenchymal transition. Whereas under control conditions, both proteins were localized mainly at cell-cell boundaries, in mesenchymal state these boundaries were dissolved and these two membrane-related proteins were more uniformly distributed throughout the cell surface, with some signal present intracellularly. The latter observation may suggest the presence of these proteins in precursory structures that give rise to exosomes (late endosomes) [9]. Thus, a direct western blot analysis confirms at least some of the changes detected in the EV proteome by mass spectrometry and points to selective enrichment of some proteins in the EV fraction, while others remain mostly cellular.

We also further explored the functional connectivity between the respective protein clusters using the String 9.0 algorithm. In this regard, induction of the mesenchymal phenotype revealed EV protein groupings around such processes as vesiculation, cell junctions and cell-ECM interactions (Fig. 5D). Overall, these results suggest that EMT-like changes induced in a 
controlled manner in a model of A431 cells lead to considerable and non-random rearrangements in the protein content of EVs these cells emit into their surroundings. 


\section{Discussion}

While the ongoing profiling efforts resulted in the assembly of valuable databases of EV-related proteins (ExoCarta; Vesclepedia [44,45], the specific processes that may influence these profiles are only beginning to emerge. In this regard EMT is of particular interest as it reflects some of the most salient and intractable features of the malignant phenotype, including cellular stemness, tumor initiating ability, invasion and metastasis $[1-3,46]$. This is also borne out in our findings, which suggest that mesenchymal A431 cells are more metastatic [8] and are enriched in the CD44-high/CD24-low phenotype, often regarded as a marker of epithelial cancer stem cells [2].

For these reasons, questions as to the vesicular representations of these biological changes in the cancer microenvironment and in patient biofluids may be highly relevant. In fact, knowledge of EMT-signatures associated with EVs could lead to development of the respective biomarker platforms, while the molecular effectors contained in such EVs may also play important functional roles in specific cancers, and represent new therapeutic targets $[15,47,48]$. Our study represents an early exploration of these possibilities, initially in a well-controlled and characterized cellular in vitro model of the mesenchymal transition in A431 cells.

In this regard it should be mentioned that several different pathways may lead to adoption of the operationally similar mesenchymal phenotype, by cells that are otherwise significantly different [1]. Therefore, while the changes we observed in the A431 model may be informative, they may not be universally applicable, and the related EV-associated EMT signatures should probably be sought in a more context-oriented manner, and with some consideration for the inducing mechanism. Thus, events acting upstream of E-cadherin may provoke EMT, either by general 
reprogramming of the cellular signalling network through oncogenic transformation e.g. upon expression of mutant Ras(Tauro et al., 2013), or by activation of EMT-inducing transcription factors, such as Twist, Snail or YB1 [49], or by direct targeting of E-cadherin expression and function [2], each with implicitly different molecular consequences. It is interesting to note, however, that amongst those diverse molecular changes the analysis of specific pathways may enable identification of common events, including in the cargo of related (e.g. adhesion, motility). Indeed, our data support this suggestion.

The biological significance of changes in the EV cargo remains to be elucidated, and may be different for cells that emit EVs and their surrounding cell, potential EV recipients [9]. For example, we demonstrated earlier that A431 cells stimulated to undergo EMT-like changes become more metastatic [8]. In this regard, the role of EVs in metastasis is increasingly well documented $[15,20,47,50-52]$. In a recent study Peinado et al presented a compelling evidence that a part of this process involves transfer of the activated MET receptor from aggressive melanoma cells (donors) to bone marrow derived regulatory cells (BMDCs), the latter of which then control formation of pre-metastatic niches in distant organs [15]. Interestingly, MET is amongst the most potent effectors of EMT [1,53], the features of which have also been observed in melanoma [54]. Indeed, intercellular trafficking of MET during melanoma metastasis could exemplify wider linkages between processes of EMT and vesiculation.

A431 cells are driven by EGFR and their expression and activity of MET is relatively low (data not shown). Therefore, the link between mesenchymal features, vesiculation and metastasis in this case is likely to entail an alternative mechanism. Indeed, activation of EGFR coupled with 
blockade of E-cadherin is sufficient to enforce mesenchymal transition in this cellular context. We have previously documented that these changes result in upregulation of the procoagulant receptor tissue factor (TF) and the onset of exaggerated procoagulant activity of both A431 cells and their EVs [9]. This is thought provoking, as the coagulation system plays a central role in metastasis $[55,56]$, and the release of procoagulant EVs into the blood stream often correlates with disease outcomes [57]. While these are intriguing possibilities, EGFR or TF are not dominant proteins in the cargo of EV emanating from mesenchymal A431 cells, which raises questions as to the identity, composition and putative functional potential of the wider EV proteome.

These questions motivated our present study, which documents several novel observations. Thus, we describe a link between epithelial-mesenchymal switching in the cancer cell phenotype and the composition of the related EV proteome. In this regard our Ingenuity analysis showed an increased number of proteins involved in EMT-linked pathways, that is: Cell-to-Cell Signaling and Interaction, Cellular Movement and Cell Morphology, in EVs emanating from mesenchymal A431 cells, as compared to controls. It is particularly striking that this difference was less pronounced in the cellular proteome than in the proteome of corresponding EVs (Fig. 2C-D). The significance of this observation requires further scrutiny, but it is tempting to speculate that the EV-related proteome may act as a highly sensitive indicator of EMT in cancer cells.

Transition of A431 cells to a mesenchymal state correlates with an enrichment of their EVs in exosomal markers CD9 and Alix (PDCD6IP), which are near the top of the list of upregulated 
proteins (Table 2). This is consistent with our recent observation based on nanoparticle tracking analysis (NTA), which suggests that mesenchymal cells produce increased amounts of exosomesize particles [9]. This observation is important as changes may occur not only with regards to packaging proteins into a specific type of EVs, e.g. exosomes, but also in the composition of EVs being produced by a given cell. We observed that A431 release vesicular structures of markedly diverse sizes (Fig. 1), and possibly with variable properties and biogenetic origin. How EMT may impact formation of exosomes, ectosomes and other forms of vesiculation remains presently unknown.

Proteome studies enable a wider assessment of molecular pathways that may converge upon certain biological phenotypes, functions or processes. This is important as the role of EVs in various tumor contexts is not identical [23]. For example, while other studies pointed to the presence of MET in the EV cargo, in our experiments a more prominent feature of EVs was a noticeable proportion of proteins involved in cell-ECM communication, especially proteins involved in integrin signalling. Similarly, results indicating the presence of integrin alphaIIb (CD41) in EVs were reported in the case of platelets, and linked to transfer of the metastatic phenotype to lung cancer cells [58].

Our proteomic analysis also showed an increased release of proteases in the EV fraction of A431 cells treated with SHE78-7 and TGF $\alpha$. These enzymes include ADAM10, (2.8 fold change) and MMP14 (2 fold). The significance of their vesicular release is presently unknown, but exosome related proteases were recently implicated in tumor cell invasion [25]. MMP-14 and integrin $\beta 1$ 
have also been found in the exosomal fraction of human fibrosarcoma (HT-1080) and melanoma (G361) cells, both endowed with metastatic potential. Interestingly, transfer of these exosomes to recipient cells stimulated other MMP activities, potentially impacting a larger microenvironment [59]. More recently, Tauro et al demonstrated, in the H-ras induced EMT model of MDCK cells, a similar increase in the presence of MMP-14, integrin $\beta 1$ and integrin $\alpha 3$ [11]. Likewise, another study documented a crucial role of integrins during EMT induction by exposure of mammary epithelial cells to TGF- $\beta 1$, and this change was implicated in tumor invasiveness [60]. Interestingly, 5 integrins upregulated in this latter context were also found at increased levels in the cargo of EVs emitted by mesenchymal A431 cells in our study (Table 2).

Interestingly, an independent analysis of the A431 proteome conducted by Park et al suggested an involvement of exosomal proteins in the metastatic potential of these cells, especially under hypoxic conditions (Park et al., 2010). EGFR and hypoxia have also been found to cooperate in induction of EMT in A431 cells [61], an observation that may explain . the focal presence of vimentin expressing cancer cells in A431 xenografts, and in human cancer samples, as we reported earlier [9]. It would be of interest to test whether EMT protein (and mRNA) signatures in circulating EVs may reflect the hypoxic nature of the underlying tumors. In line with this supposition, the protein profiles of hypoxic A431 cells published recently by Park etl, reveal similar classes of changes as those we observed in EVs upon EMT induction using TGF $\alpha$ and SHE78-7 antibody, including emission of proteins linked to cell-cell contact and cell-ECM adhesion [62]. 
Thus, our study documents for the first time the scope of proteomic changes that are induced in EVs of epithelial cancer cells in the course of mesenchymal changes imposed in a manner dependent on the activity of EGFR and E-cadherin. We believe that these results add new perspective to the previous proteomic studies performed on EV fractions [63-65], by demonstrating the molecular plasticity of the vesiculation process and its links to cellular differentiation and transformation. What remains to be determined is the functional consequence

of the emission, retention and/or intercellular transfer of proteins we identified in the cargo of EVs. We can only infer this from the nature of the molecular composition of tumor-derived EVs, and more direct approaches are to follow. This question is especially relevant for processes such as metastasis, where both EMT and vesiculation play increasingly prominent roles [11].

\section{Conflict of interest}

Authors declare no conflict of interest

\section{Acknowledgements}

This work was supported by grants to J.R. from the Canadian Institutes of Health Research (CIHR, MOP 102736 and MOP 111119). DG is a recipient of Thelma Adams doctoral fellowhip from the MCH Foundation. Also Fellowship support (NM) and infrastructure was from Fonds de la recherche en santé du Québec (FRSQ). JR is the Jack Cole Chair in Pediatric Oncology. We thank our colleagues for helpful discussions. 


\section{Figure legends}

Figure 1 - A431 epithelial cancer cells produce extracellular vesicles (EVs). A. Overview of reported changes in A431 vesiculation upon induction of the mesenchymal phenotype by blockade of E-cadherin (SHE78-7) and stimulation of EGFR in the presence of TGF $\alpha$ (see text). B-C. Morphology of A431 cells in their epithelial and mesenchymal states, observed by confocal microscopy after staining of F-actin with phalloidin. D. Electron microscopy of the heterogeneous EV population produced by A431 cells. E-G. EMT-related enrichment of A431 cells in the immunophenotype of cancer stem cells (CD44-high/CD24-low) - FACS analysis. Forward versus site scatter analysis (E); Dot plot analysis documenting heterogeneity and phenotypic shift of cells stained for CD44 and CD24 (F). Quantification of CD44/CD24 phenotypes in control and EMT populations of A431 cells (G).

Figure 2 - Overview of the type and function of proteins detected by MS in A431 extracellular vesicles (EVs). Classification of protein content of A431 EVs, according to the subcellular compartment they are derived from (A), the biological process they are involved in (B), their molecular function (C) and protein class (D).

Figure 3 - The transition to the mesenchymal state correlates with EVs changes characteristic of EMT. Venn diagrams illustrating the overlap of proteins detected in A431 cells (A) and in their corresponding EVs (B), in control conditions, after treatment with SHE78-7 antibody, TGF $\alpha$ or the two agents combined. Treatment-related changes in cells (C) and EV (D) proteomes were classified according to biological functions (IPA Software), four of them are shown. Of note is 
the greatest magnitude of changes induced by double treatment and the link between these responses and processes involved in invasion and metastasis (cellular movement, morphology).

Figure 4 - Graphic representation of the differential protein expression profiles after induction of mesenchymal state in A431 cells. The heatmap represents proteins for which an increase (red) or decrease (green) was observed in A431 cells or corresponding EVs after treatment (SHE78-7, TGF represents proteins fo), compared to control conditions. Only proteins for which there was more than 4 fold increase or decrease in expression compared to the control cells or EVs were included. Grey boxes refer to absence of detection of the corresponding protein. Of note are the differences between cells and their corresponding EVs, and between different treatments.

Figure 5 - Validation of selected protein alterations in cells and EVs of A431 cells in their epithelial and mesenchymal states. A. Western-blot analysis of A431 cells and their corresponding EVs documents differential expression of integrin- $\alpha 2, \gamma$-catenin and CD9, while GAPDH was used as a loading control. Immunofluorescent staining for integrin- $\alpha 2$ (B) and $\gamma$ catenin (C) visualizes membrane location of these two proteins. D. Network analysis with String 9.0 software reveales functional connections between 17 of the 32 proteins upregulated by SHE78-7/TGF $\alpha$ treatment. (*: proteins upregulated in our datasets, other proteins are shown to succinctly describe the pathways involved).

Table 1 - List of the 25 most abundant proteins in A431 EVs. 
Selection of the 25 most abundant proteins identified in EVs secreted by A431 cells in the extracellular media, according to the total spectral counts.

Table 2 - Listing of 32 proteins upregulated by more than 2 fold in EVs from A431 cells in their mesenchymal state. Proteins for which the spectral count documented enrichment in the cargo of EVs emitted by SHE78-7/TGF $\alpha$-treated cells were compared to their representation in the cargo of EVs from control cells. For 32 such proteins the EMT-dependent induction was greater than 2 fold. 


\section{References}

1. J.P.Thiery, H.Acloque, R.Y.Huang, M.A.Nieto (2009). Epithelial-mesenchymal transitions in development and disease. Cell. 139, 871-890.

2. S.A.Mani, W.Guo, M.J.Liao, E.N.Eaton, A.Ayyanan, A.Y.Zhou, M.Brooks, F.Reinhard, C.C.Zhang, M.Shipitsin, L.L.Campbell, K.Polyak, C.Brisken, J.Yang, R.A.Weinberg (2008). The epithelial-mesenchymal transition generates cells with properties of stem cells. Cell 133, 704-715.

3. Y.Liu, Y.Zhou, K.Zhu (2012). Inhibition of glioma cell lysosome exocytosis inhibits glioma invasion. PLoS.ONE. 7, e45910.

4. K.Polyak, R.A.Weinberg (2009). Transitions between epithelial and mesenchymal states: acquisition of malignant and stem cell traits. Nat.Rev.Cancer. 9, 265-273.

5. N.Tiwari, A.Gheldof, M.Tatari, G.Christofori (2012). EMT as the ultimate survival mechanism of cancer cells. Semin. Cancer Biol. 22, 194-207.

6. C.B.St, C.Sheehan, J.W.Rak, V.A.Florenes, J.M.Slingerland, R.S.Kerbel (1998). ECadherin-dependent growth suppression is mediated by the cyclin-dependent kinase inhibitor p27(KIP1). J.Cell Biol. 142, 557-571.

7. T.T.Onder, P.B.Gupta, S.A.Mani, J.Yang, E.S.Lander, R.A.Weinberg (2008). Loss of Ecadherin promotes metastasis via multiple downstream transcriptional pathways. Cancer Res. 68, 3645-3654.

8. C.C.Milsom, J.L.Yu, N.Mackman, J.Micallef, G.M.Anderson, A.Guha, J.W.Rak (2008). Tissue factor regulation by epidermal growth factor receptor and epithelial-tomesenchymal transitions: effect on tumor initiation and angiogenesis. Cancer Res. 68, 10068-10076.

9. D.Garnier, N.Magnus, T.H.Lee, V.Bentley, B.Meehan, C.Milsom, L.Montermini, T.Kislinger, J.Rak (2012). Cancer Cells Induced to Express Mesenchymal Phenotype Release Exosome-like Extracellular Vesicles Carrying Tissue Factor. J Biol.Chem. 287, 43565-43572.

10. V.Luga, L.Zhang, A.M.Viloria-Petit, A.A.Ogunjimi, M.R.Inanlou, E.Chiu, M.Buchanan, A.N.Hosein, M.Basik, J.L.Wrana (2012). Exosomes Mediate Stromal Mobilization of Autocrine Wnt-PCP Signaling in Breast Cancer Cell Migration. Cell. 151, 1542-1556.

11. B.J.Tauro, R.A.Mathias, D.W.Greening, S.K.Gopal, H.Ji, E.A.Kapp, B.M.Coleman, A.F.Hill, U.Kusebauch, J.L.Hallows, D.Shteynberg, R.L.Moritz, H.J.Zhu, R.J.Simpson (2013). Oncogenic H-Ras reprograms Madin-Darby canine kidney (MDCK) cell-derived exosomal proteins following epithelial-mesenchymal transition. Mol.Cell Proteomics. 
12. T.H.Lee, E.D'Asti, N.Magnus, K.Al-Nedawi, B.Meehan, J.Rak (2011). Microvesicles as mediators of intercellular communication in cancer-the emerging science of cellular 'debris'. Semin.Immunopathol.

13. C.Thery, M.Ostrowski, E.Segura (2009). Membrane vesicles as conveyors of immune responses. Nat.Rev.Immunol. 9, 581-593.

14. K.Al-Nedawi, B.Meehan, J.Micallef, V.Lhotak, L.May, A.Guha, J.Rak (2008). Intercellular transfer of the oncogenic receptor EGFRvIII by microvesicles derived from tumour cells. Nat.Cell Biol. 10, 619-624.

15. H.Peinado, M.Aleckovic, S.Lavotshkin, I.Matei, B.Costa-Silva, G.Moreno-Bueno, M.Hergueta-Redondo, C.Williams, G.Garcia-Santos, C.M.Ghajar, A.Nitadori-Hoshino, C.Hoffman, K.Badal, B.A.Garcia, M.K.Callahan, J.Yuan, V.R.Martins, J.Skog, R.N.Kaplan, M.S.Brady, J.D.Wolchok, P.B.Chapman, Y.Kang, J.Bromberg, D.Lyden (2012). Melanoma exosomes educate bone marrow progenitor cells toward a prometastatic phenotype through MET. Nat.Med. 18, 833-891.

16. J.L.Hood, H.Pan, G.M.Lanza, S.A.Wickline (2009). Paracrine induction of endothelium by tumor exosomes. Lab Invest. 89, 1317-1328.

17. M.A.Antonyak, B.Li, L.K.Boroughs, J.L.Johnson, J.E.Druso, K.L.Bryant, D.A.Holowka, R.A.Cerione (2011). Cancer cell-derived microvesicles induce transformation by transferring tissue transglutaminase and fibronectin to recipient cells. Proc.Natl.Acad.Sci.U.S.A. 108, 4852-4857.

18. M.C.Deregibus, V.Cantaluppi, R.Calogero, I.M.Lo, C.Tetta, L.Biancone, S.Bruno, B.Bussolati, G.Camussi (2007). Endothelial progenitor cell derived microvesicles activate an angiogenic program in endothelial cells by a horizontal transfer of mRNA. Blood. 110, 2440-2448.

19. J.Ratajczak, M.Wysoczynski, F.Hayek, A.Janowska-Wieczorek, M.Z.Ratajczak (2006). Membrane-derived microvesicles: important and underappreciated mediators of cell-tocell communication. Leukemia 20, 1487-1495.

20. A.Bobrie, S.Krumeich, F.Reyal, C.Recchi, L.F.Moita, M.C.Seabra, M.Ostrowski, C.Thery (2012). Rab27a supports exosome-dependent and -independent mechanisms that modify the tumor microenvironment and can promote tumor progression. Cancer Res. 72, 4920-4930.

21. J.Skog, T.Wurdinger, R.S.van, D.H.Meijer, L.Gainche, W.T.Curry, Jr., B.S.Carter, A.M.Krichevsky, X.O.Breakefield (2008). Glioblastoma microvesicles transport RNA and proteins that promote tumour growth and provide diagnostic biomarkers. Nat.Cell Biol 10, 1470-1476.

22. T.B.Russell, A.M.Skinner, P.Kurre (2012). Programmed vesicle transfer of green fluorescent protein from a stably transduced cell line to primary hematopoietic cells. Blood. 119, 5330-5332. 
23. A.Bobrie, M.Colombo, G.Raposo, C.Thery (2011). Exosome secretion: molecular mechanisms and roles in immune responses. Traffic. 12, 1659-1668.

24. R.B.Koumangoye, A.M.Sakwe, J.S.Goodwin, T.Patel, J.Ochieng (2011). Detachment of breast tumor cells induces rapid secretion of exosomes which subsequently mediate cellular adhesion and spreading. PLoS.ONE. 6, e24234.

25. A.Hendrix, W.Westbroek, M.Bracke, W.O.De (2010). An ex(o)citing machinery for invasive tumor growth. Cancer Res. 70, 9533-9537.

26. R.M.Johnstone (2006). Exosomes biological significance: A concise review. Blood Cells Mol.Dis. 36, 315-321.

27. A.Chairoungdua, D.L.Smith, P.Pochard, M.Hull, M.J.Caplan (2010). Exosome release of beta-catenin: a novel mechanism that antagonizes Wnt signaling. J.Cell Biol. \%20;190, 1079-1091.

28. H.Sheldon, E.Heikamp, H.Turley, R.Dragovic, P.Thomas, C.E.Oon, R.Leek, M.Edelmann, B.Kessler, R.C.Sainson, I.Sargent, J.L.Li, A.L.Harris (2010). New mechanism for Notch signaling to endothelium at a distance by Delta-like 4 incorporation into exosomes. Blood. 116, 2385-2394.

29. F.J.Verweij, J.M.Middeldorp, D.M.Pegtel (2012). Intracellular signaling controlled by the endosomal-exosomal pathway. Commun.Integr.Biol. 5, 88-93.

30. J.C.Gross, V.Chaudhary, K.Bartscherer, M.Boutros (2012). Active Wnt proteins are secreted on exosomes. Nat.Cell Biol. 14, 1036-1045.

31. M.F.Bolukbasi, A.Mizrak, G.B.Ozdener, S.Madlener, T.Strobel, E.P.Erkan, J.B.Fan, X.O.Breakefield, O.Saydam (2012). miR-1289 and "Zipcode"-like Sequence Enrich mRNAs in Microvesicles. Mol.Ther.Nucleic Acids. 1:e10. doi: 10.1038/mtna.2011.2., e10.

32. H.Valadi, K.Ekstrom, A.Bossios, M.Sjostrand, J.J.Lee, J.O.Lotvall (2007). Exosomemediated transfer of mRNAs and microRNAs is a novel mechanism of genetic exchange between cells. Nat.Cell Biol. 9, 654-659.

33. J.Yu, L.May, C.Milsom, G.M.Anderson, J.I.Weitz, J.P.Luyendyk, G.Broze, N.Mackman, J.Rak (2008). Contribution of host-derived tissue factor to tumor neovascularization. Arterioscler.Thromb.Vasc.Biol 28, 1975-1981.

34. F.Bianco, C.Perrotta, L.Novellino, M.Francolini, L.Riganti, E.Menna, L.Saglietti, E.H.Schuchman, R.Furlan, E.Clementi, M.Matteoli, C.Verderio (2009). Acid sphingomyelinase activity triggers microparticle release from glial cells. EMBO J. $\mathbf{2 8}$, 1043-1054. 
35. N.Magnus, D.Garnier, J.Rak (2010). Oncogenic epidermal growth factor receptor upregulates multiple elements of the tissue factor signaling pathway in human glioma cells. Blood. 116, 815-818.

36. K.L.Sodek, A.I.Evangelou, A.Ignatchenko, M.Agochiya, T.J.Brown, M.J.Ringuette, I.Jurisica, T.Kislinger (2008). Identification of pathways associated with invasive behavior by ovarian cancer cells using multidimensional protein identification technology (MudPIT). Mol.Biosyst. 4, 762-773.

37. R.Craig, R.C.Beavis (2004). TANDEM: matching proteins with tandem mass spectra. Bioinformatics. 20, 1466-1467.

38. T.Kislinger, B.Cox, A.Kannan, C.Chung, P.Hu, A.Ignatchenko, M.S.Scott, A.O.Gramolini, Q.Morris, M.T.Hallett, J.Rossant, T.R.Hughes, B.Frey, A.Emili (2006). Global survey of organ and organelle protein expression in mouse: combined proteomic and transcriptomic profiling. Cell. 125, 173-186.

39. H.Liu, R.G.Sadygov, J.R.Yates, III (2004). A model for random sampling and estimation of relative protein abundance in shotgun proteomics. Anal.Chem. 76, 4193-4201.

40. L.L.Marotta, V.Almendro, A.Marusyk, M.Shipitsin, J.Schemme, S.R.Walker, N.Bloushtain-Qimron, J.J.Kim, S.A.Choudhury, R.Maruyama, Z.Wu, M.Gonen, L.A.Mulvey, M.O.Bessarabova, S.J.Huh, S.J.Silver, S.Y.Kim, S.Y.Park, H.E.Lee, K.S.Anderson, A.L.Richardson, T.Nikolskaya, Y.Nikolsky, X.S.Liu, D.E.Root, W.C.Hahn, D.A.Frank, K.Polyak (2011). The JAK2/STAT3 signaling pathway is required for growth of CD44(+)CD24(-) stem cell-like breast cancer cells in human tumors. J Clin Invest. 121, 2723-2735.

41. C.Oliveras-Ferraros, A.Vazquez-Martin, S.Cufi, B.Queralt, L.Baez, R.Guardeno, X.Hernandez-Yague, B.Martin-Castillo, J.Brunet, J.A.Menendez (2011). Stem cell property epithelial-to-mesenchymal transition is a core transcriptional network for predicting cetuximab (Erbitux) efficacy in KRAS wild-type tumor cells. J Cell Biochem. 112, 10-29.

42. S.Geng, Y.Guo, Q.Wang, L.Li, J.Wang (2013). Cancer stem-like cells enriched with CD29 and CD44 markers exhibit molecular characteristics with epithelial-mesenchymal transition in squamous cell carcinoma. Arch.Dermatol.Res. 305, 35-47.

43. A.Bobrie, C.Thery (2013). Exosomes and communication between tumours and the immune system: are all exosomes equal? Biochem.Soc.Trans. 41, 263-267.

44. S.Mathivanan, C.J.Fahner, G.E.Reid, R.J.Simpson (2011). ExoCarta 2012: database of exosomal proteins, RNA and lipids. Nucleic Acids Res.

45. H.Kalra, R.J.Simpson, H.Ji, E.Aikawa, P.Altevogt, P.Askenase, V.C.Bond, F.E.Borras, X.Breakefield, V.Budnik, E.Buzas, G.Camussi, A.Clayton, E.Cocucci, J.M.Falcon-Perez, S.Gabrielsson, Y.S.Gho, D.Gupta, H.C.Harsha, A.Hendrix, A.F.Hill, J.M.Inal, G.Jenster, E.M.Kramer-Albers, S.K.Lim, A.Llorente, J.Lotvall, A.Marcilla, L.Mincheva-Nilsson, 
I.Nazarenko, R.Nieuwland, E.N.Nolte-'t Hoen, A.Pandey, T.Patel, M.G.Piper, S.Pluchino, T.S.Prasad, L.Rajendran, G.Raposo, M.Record, G.E.Reid, F.SanchezMadrid, R.M.Schiffelers, P.Siljander, A.Stensballe, W.Stoorvogel, D.Taylor, C.Thery, H.Valadi, B.W.van Balkom, J.Vazquez, M.Vidal, M.H.Wauben, M.Yanez-Mo, M.Zoeller, S.Mathivanan (2012). Vesiclepedia: a compendium for extracellular vesicles with continuous community annotation. PLoS.Biol. 10, e1001450.

46. T.Brabletz, A.Jung, S.Spaderna, F.Hlubek, T.Kirchner (2005). Opinion: migrating cancer stem cells - an integrated concept of malignant tumour progression. Nat.Rev.Cancer $\mathbf{5}$, 744-749.

47. J.L.Hood, R.S.San, S.A.Wickline (2011). Exosomes released by melanoma cells prepare sentinel lymph nodes for tumor metastasis. Cancer Res. 71, 3792-3801.

48. H.Shao, J.Chung, L.Balaj, A.Charest, D.D.Bigner, B.S.Carter, F.H.Hochberg, X.O.Breakefield, R.Weissleder, H.Lee (2012). Protein typing of circulating microvesicles allows real-time monitoring of glioblastoma therapy. Journal of Extracellular Vesicles $\mathbf{1}$, 26.

49. R.Kalluri, R.A.Weinberg (2009). The basics of epithelial-mesenchymal transition. J Clin Invest. 119, 1420-1428.

50. T.Jung, D.Castellana, P.Klingbeil, H.Cuesta, I, M.Vitacolonna, D.J.Orlicky, S.R.Roffler, P.Brodt, M.Zoller (2009). CD44v6 dependence of premetastatic niche preparation by exosomes. Neoplasia. 11, 1093-1105.

51. G.Poste, G.L.Nicolson (1980). Arrest and metastasis of blood-borne tumor cells are modified by fusion of plasma membrane vesicles from highly metastatic cells. Proc.Natl.Acad.Sci.U.S.A. 77, 399-403.

52. S.Hao, Z.Ye, F.Li, Q.Meng, M.Qureshi, J.Yang, J.Xiang (2006). Epigenetic transfer of metastatic activity by uptake of highly metastatic B16 melanoma cell-released exosomes. Exp.Oncol. 28, 126-131.

53. S.Pennacchietti, P.Michieli, M.Galluzzo, M.Mazzone, S.Giordano, P.M.Comoglio (2003). Hypoxia promotes invasive growth by transcriptional activation of the met protooncogene. Cancer Cell 3, 347-361.

54. K.Lin, S.Baritaki, L.Militello, G.Malaponte, Y.Bevelacqua, B.Bonavida (2010). The Role of B-RAF Mutations in Melanoma and the Induction of EMT via Dysregulation of the NF-kappaB/Snail/RKIP/PTEN Circuit. Genes Cancer. 1, 409-420.

55. J.S.Palumbo, K.E.Talmage, J.V.Massari, C.M.La Jeunesse, M.J.Flick, K.W.Kombrinck, Z.Hu, K.A.Barney, J.L.Degen (2007). Tumor cell-associated tissue factor and circulating hemostatic factors cooperate to increase metastatic potential through natural killer celldependent and-independent mechanisms. Blood 110, 133-141. 
56. H.H.Versteeg, F.Schaffner, M.Kerver, H.H.Petersen, J.Ahamed, B.Felding-Habermann, Y.Takada, B.M.Mueller, W.Ruf (2008). Inhibition of tissue factor signaling suppresses tumor growth. Blood 111, 190-199.

57. J.Thaler, C.Ay, N.Mackman, R.M.Bertina, A.Kaider, C.Marosi, N.S.Key, D.A.Barcel, W.Scheithauer, G.Kornek, C.Zielinski, I.Pabinger (2012). Microparticle-associated tissue factor activity, venous thromboembolism and mortality in pancreatic, gastric, colorectal and brain cancer patients. J Thromb.Haemost. 10, 1363-1370.

58. A.Janowska-Wieczorek, M.Wysoczynski, J.Kijowski, L.Marquez-Curtis, B.Machalinski, J.Ratajczak, M.Z.Ratajczak (2005). Microvesicles derived from activated platelets induce metastasis and angiogenesis in lung cancer. Int.J.Cancer 113, 752-760.

59. J.Hakulinen, L.Sankkila, N.Sugiyama, K.Lehti, J.Keski-Oja (2008). Secretion of active membrane type 1 matrix metalloproteinase (MMP-14) into extracellular space in microvesicular exosomes. J Cell Biochem. 105, 1211-1218.

60. S.Maschler, G.Wirl, H.Spring, D.V.Bredow, I.Sordat, H.Beug, E.Reichmann (2005). Tumor cell invasiveness correlates with changes in integrin expression and localization. Oncogene. 24, 2032-2041.

61. A.Misra, C.Pandey, S.K.Sze, T.Thanabalu (2012). Hypoxia activated EGFR signaling induces epithelial to mesenchymal transition (EMT). PLoS.ONE. 7, e49766.

62. J.E.Park, H.S.Tan, A.Datta, R.C.Lai, H.Zhang, W.Meng, S.K.Lim, S.K.Sze (2010). Hypoxic tumor cell modulates its microenvironment to enhance angiogenic and metastatic potential by secretion of proteins and exosomes. Mol.Cell Proteomics. 9, 1085-1099.

63. F.Simona, S.Laura, T.Simona, A.Riccardo (2013). Contribution of proteomics to understanding the role of tumor-derived exosomes in cancer progression: State of the art and new perspectives. Proteomics. 13, 1581-1594.

64. R.J.Simpson, J.W.Lim, R.L.Moritz, S.Mathivanan (2009). Exosomes: proteomic insights and diagnostic potential. Expert.Rev.Proteomics. 6, 267-283.

65. M.C.Henderson, D.O.Azorsa (2012). The genomic and proteomic content of cancer cellderived exosomes. Front Oncol. 2:38. Epub;2012 Apr 17., 38. 
A
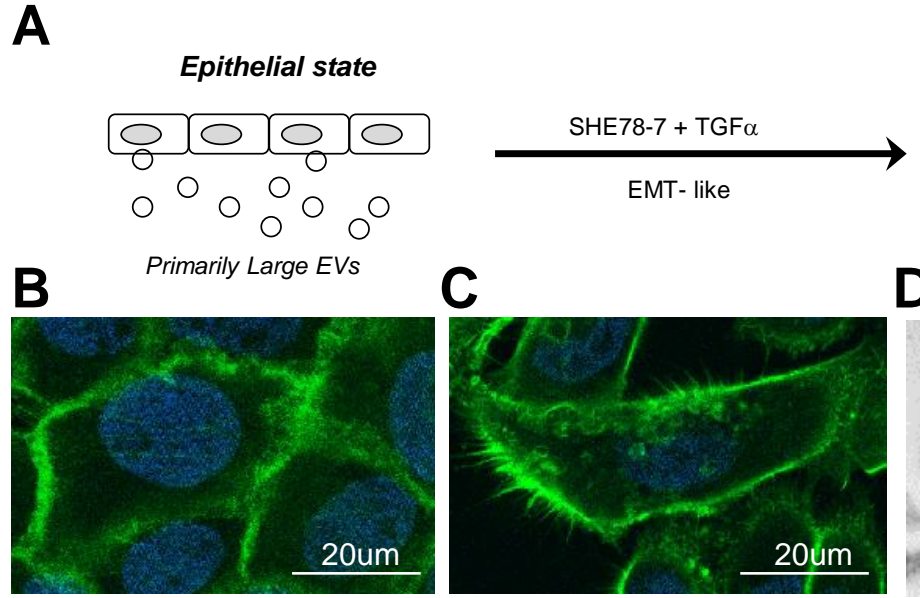

D Exosome-like EVs +Large EVs
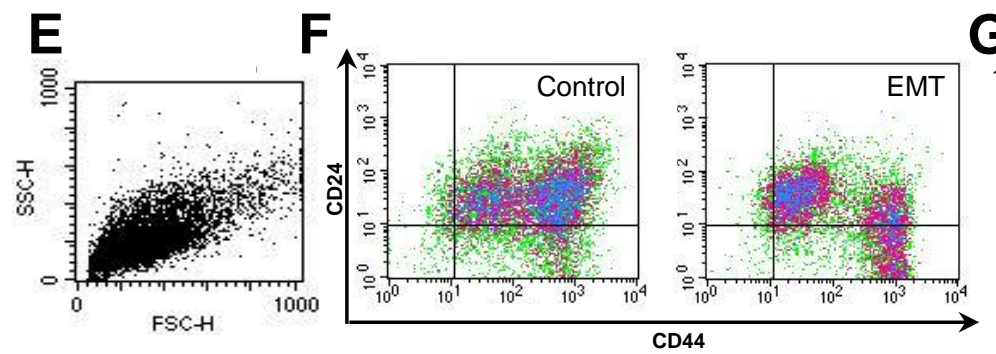

G

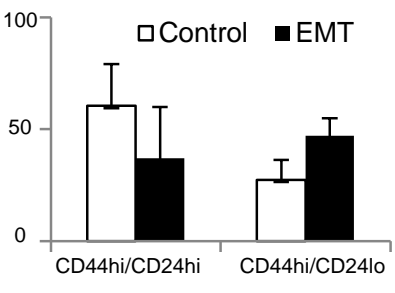

Figure 1 


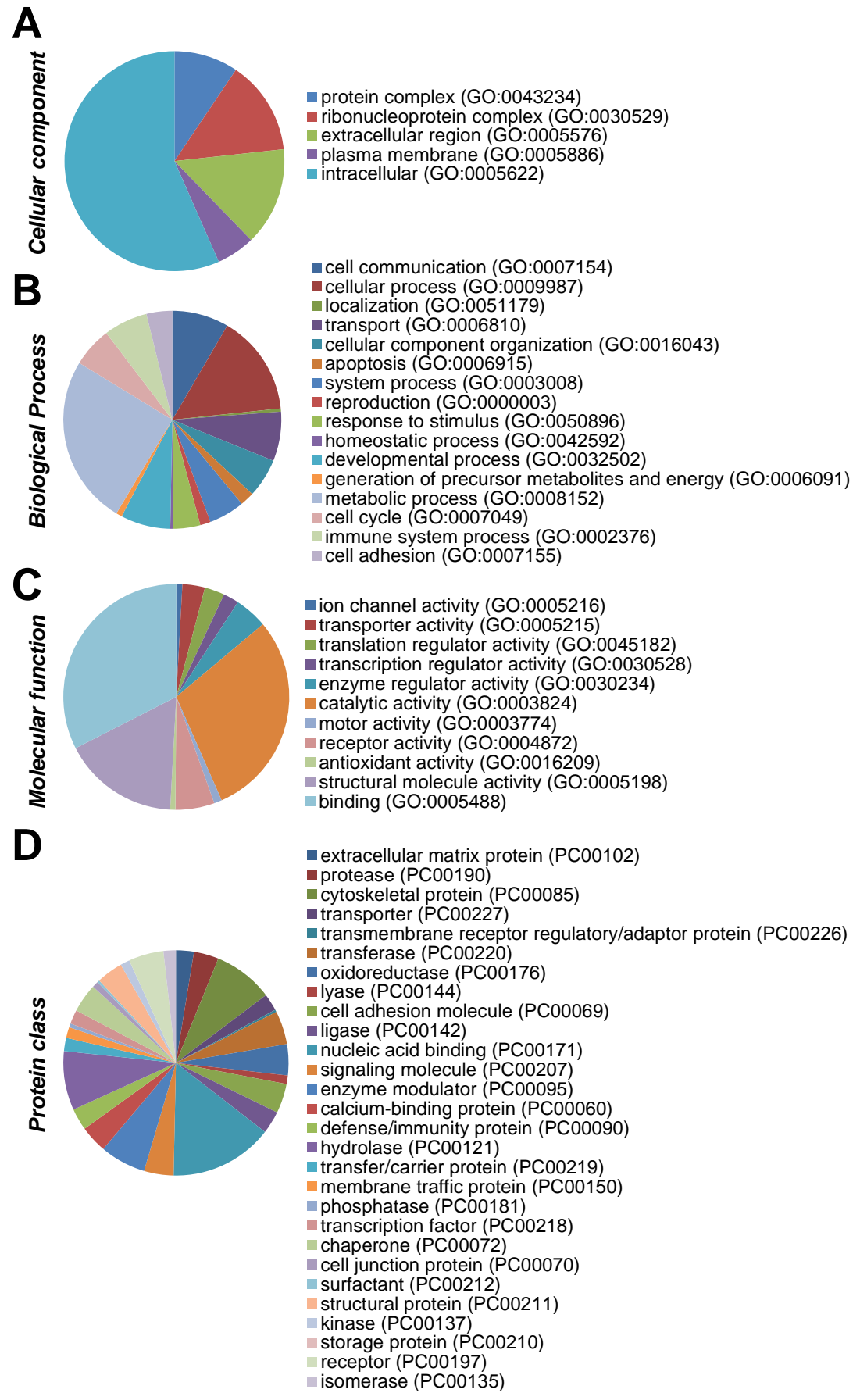

Figure 2 


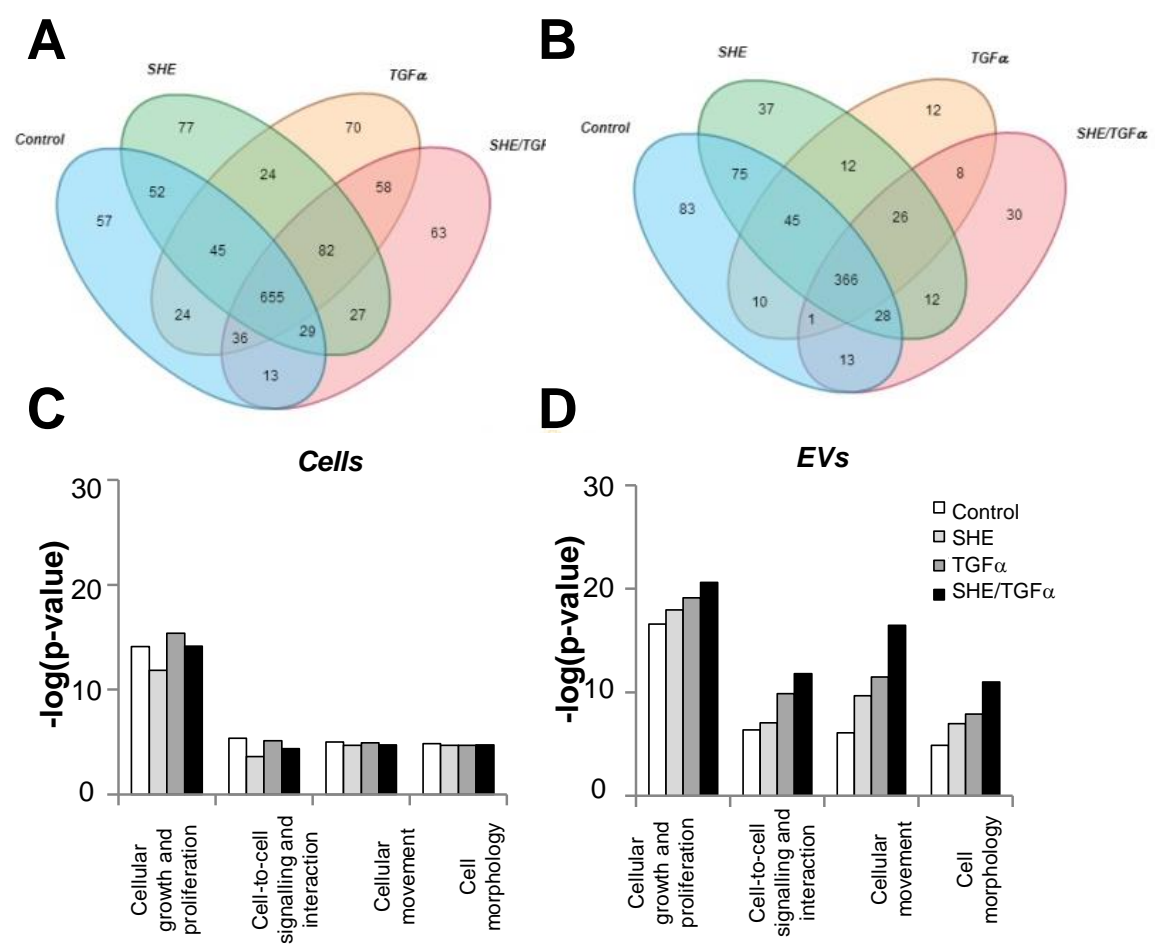

Figure 3 


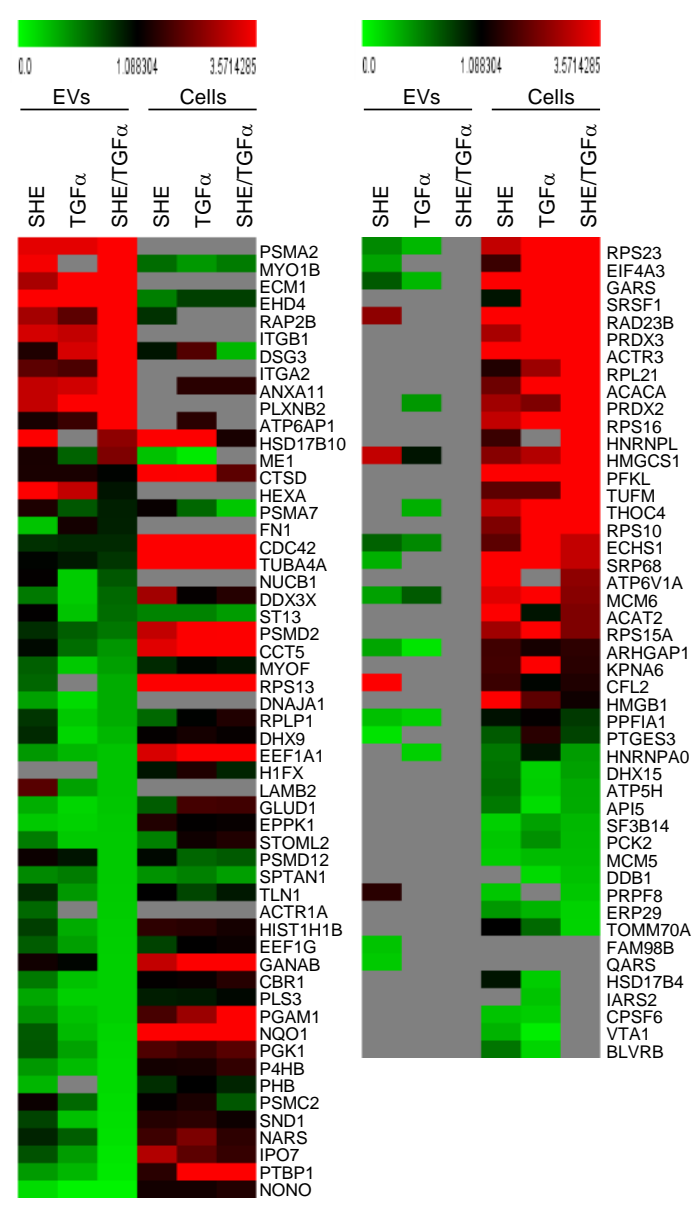

Figure 4 
A
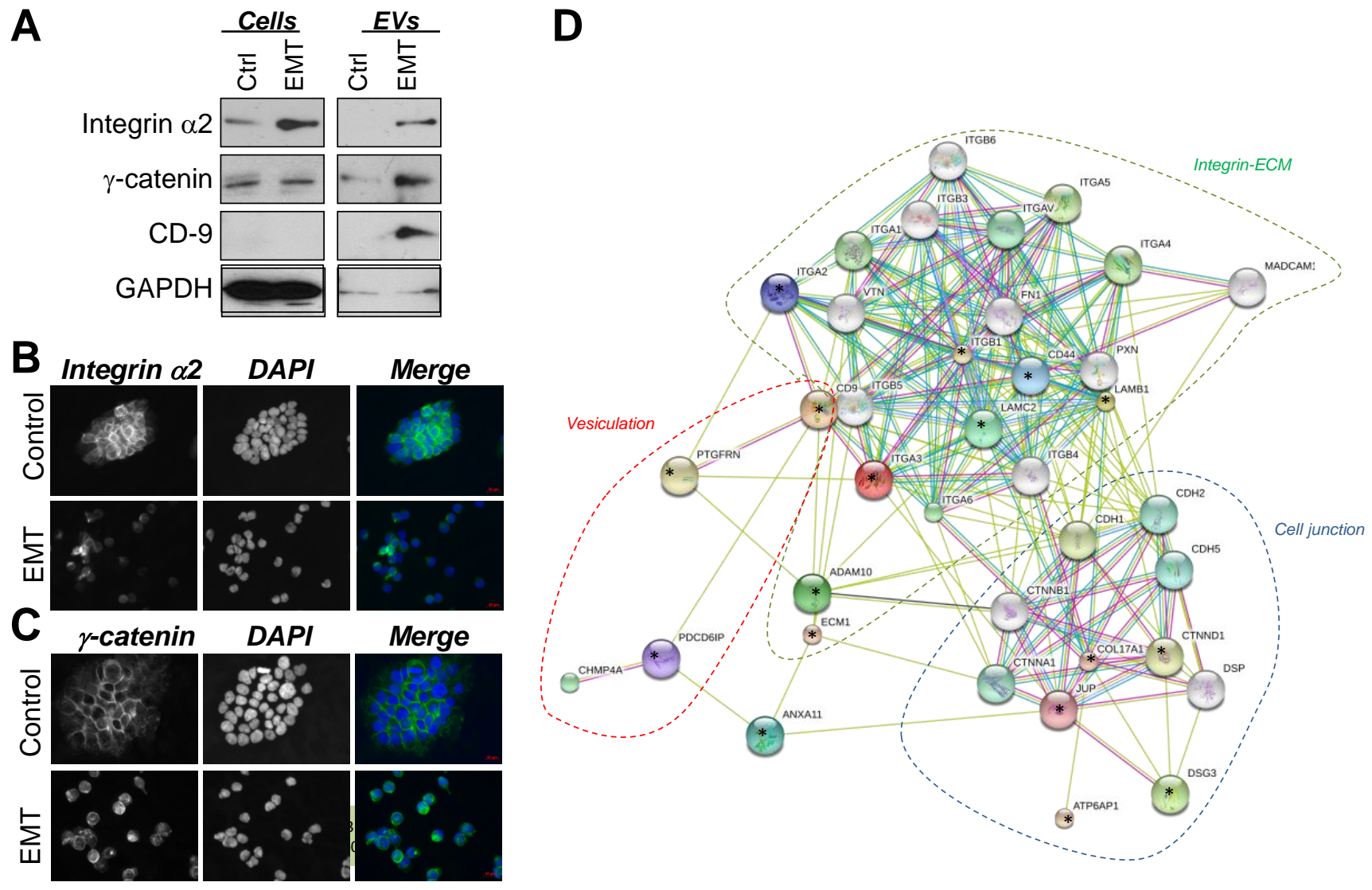

Figure 5 
Table 1 - List of the 25 most abundant proteins in A431 EVs.

\begin{tabular}{l|l}
\hline FASN & Fatty acid synthase \\
EEF1A1 & Elongation factor 1-alpha 1 \\
HSP90AB1 & Heat shock protein HSP 90-beta \\
ANXA2 & Annexin A2 \\
ACTB & Actin, cytoplasmic 1 \\
PKM2 & Pyruvate kinase isozymes M1/M2 \\
MYH9 & Myosin-9 \\
TUBB & Tubulin beta chain \\
HSP90AA1 & Heat shock protein HSP 90-alpha \\
LGALS3BP & Galectin-3-binding protein \\
ENO1 & Alpha-enolase \\
HSPA8 & Heat shock cognate 71 kDa protein \\
TUBB2C & Tubulin beta-2C chain \\
TUBB3 & Tubulin beta-3 chain \\
CTSD & Cathepsin D \\
TUBA1B & Tubulin alpha-1B chain \\
HIST1H4A & Histone H4 \\
HIST1H2BK & Histone H2B type 1-K \\
HIST2H2AC & Histone H2A type 2-C \\
ACTN1 & Alpha-actinin-1 \\
HIST1H2BJ & Histone H2B type 1-J \\
HSPA1A & Heat shock 70 kDa protein 1A/1B \\
COL12A1 & Collagen alpha-1(XII) chain \\
HSPA5 & |78 kDa glucose-regulated protein \\
CLU & Clusterin \\
\hline
\end{tabular}

Table 2 - List of the 32 proteins upregulated more than 2 fold in EVs from A431 cells treated with SHE/TGF $\alpha$.

\begin{tabular}{l|l}
\hline PSMA2 & Proteasome subunit alpha type-2 \\
MYO1B & Myosin-lb \\
ECM1 & Extracellular matrix protein 1 \\
EHD4 & EH domain-containing protein 4 \\
RAP2B & Ras-related protein Rap-2b \\
ITGB1 & Integrin beta-1 \\
DSG3 & Desmoglein-3 \\
ITGA2 & Integrin alpha-2 \\
ANXA11 & Annexin A11 \\
ATP6AP1 & V-type proton ATPase subunit S1 \\
PLXNB2 & Plexin-B2 \\
PTGFRN & Prostaglandin F2 receptor negative regulator \\
PDCD6IP & Programmed cell death 6-interacting protein \\
COL17A1 & Collagen alpha-1(XVII) chain \\
ADAM10 & Disintegrin and metalloproteinase domain-containing protein 10 \\
RPS12 & 40S ribosomal protein S12 \\
DNAJA2 & DnaJ homolog subfamily A member 2 \\
LAMC2 & Laminin subunit gamma-2 \\
ATP6AP2 & Renin receptor \\
HSD17B10 & 3-hydroxyacyl-CoA dehydrogenase type-2 \\
ITGA3 & Integrin alpha-3 \\
JUP & Junction plakoglobin \\
TYMP & Thymidine phosphorylase \\
LAMB1 & Laminin subunit beta-1 \\
ME1 & NADP-dependent malic enzyme \\
LSR & Lipolysis-stimulated lipoprotein receptor \\
CD9 & CD9 antigen \\
SLC2A1 & Solute carrier family 2, facilitated glucose transporter member 1 \\
CTNND1 & Catenin delta-1 \\
MARCKS & Myristoylated alanine-rich C-kinase substrate \\
CD44 & CD44 antigen \\
QSOX1 & Sulfhydryl oxidase 1 \\
\hline
\end{tabular}

